\title{
Verification and Validation of a Fingerprint Image Registration Software
}

\author{
Dejan Desovski, ${ }^{1}$ Vijai Gandikota, ${ }^{1}$ Yan Liu, ${ }^{2}$ Yue Jiang, ${ }^{1}$ and Bojan Cukic ${ }^{1}$ \\ ${ }^{1}$ Lane Department of Computer Science and Electrical Engineering, West Virginia University, Morgantown, WV 26506-6109, USA \\ ${ }^{2}$ Motorola Labs, Motorola Inc., Schaumburg, IL 60196, USA
}

Received 28 February 2005; Revised 14 September 2005; Accepted 21 October 2005

The need for reliable identification and authentication is driving the increased use of biometric devices and systems. Verification and validation techniques applicable to these systems are rather immature and ad hoc, yet the consequences of the wide deployment of biometric systems could be significant. In this paper we discuss an approach towards validation and reliability estimation of a fingerprint registration software. Our validation approach includes the following three steps: (a) the validation of the source code with respect to the system requirements specification; (b) the validation of the optimization algorithm, which is in the core of the registration system; and (c) the automation of testing. Since the optimization algorithm is heuristic in nature, mathematical analysis and test results are used to estimate the reliability and perform failure analysis of the image registration module.

Copyright () 2006 Hindawi Publishing Corporation. All rights reserved.

\section{INTRODUCTION}

The application of biometric devices and systems is experiencing significant growth, primarily due to the increasing need for reliable authentication and identification [1]. For example, fingerprint identification is used at airports for securing border crossing, but also in our offices as a password replacement. Typical biometric system classifies users as genuine or imposters depending on a selected threshold. For example, if 50 is selected as a threshold for the device whose performance characteristics are depicted in Figure 1, all users with scores higher than 50 will be classified as imposters, while those with scores less than 50 will be classified as genuine. Consequently, the failures of biometric systems include false positives (an imposter classified as a genuine) and false negatives (a genuine user classified as an imposter).

Different algorithms [2,3] in biometric systems have the goal of increasing the rate of success and at the same time decreasing the rate of failure. Depending on the actual application environment, the cost impact of failures might be different. In an office setup, a rejected fingerprint (false negative) causes the user to repeat the authentication procedure. However, if a fingerprint recognition device makes a false match (false positive) in matters of national security or criminal court cases, the potential of grave consequences is obvious.

Most biometric applications (e.g., fingerprint, face, hand geometry, iris scans) work with images. An image of a biometric feature is easy to acquire. Unfortunately, studies of image processing systems in the software reliability engineering arena are rare. One of the reasons might be the enormous size of the input space. Considering a $256 \times 256$ black and white image, we have $2^{65536}$ possible inputs, excluding any possibility of achieving input space coverage during software testing. Another significant problem for evaluation of imaging algorithms is defining appropriate objective metrics, which will be indicative of the algorithm's performance. This difficulty arises because of the fact that it is hard to quantify human visual perception.

Quite surprisingly, reliability studies applied to biometric systems are rare too. The most likely reason is the unavailability of sufficiently large test data sets. Testing a biometric system involves human subjects. Therefore, publicly available datasets and data acquisition efforts must deal with related privacy issues. Consequently, commercially available biometric systems make no reliability claims and, if they do, the claims may be meaningless if based on test population sizes that do not approach statistical significance.

Our research group has been recently approached to assess the quality attributes of a fingerprint image registration software-one component of a fingerprint recognition system. In many usage scenarios, an acquired fingerprint image needs to be compared, automatically or manually, with a stored image. The images are usually misaligned, rotated or scaled, possibly containing different noise patterns due to varying image acquisition circumstances. These images need to be registered, that is, automatically aligned in the same 


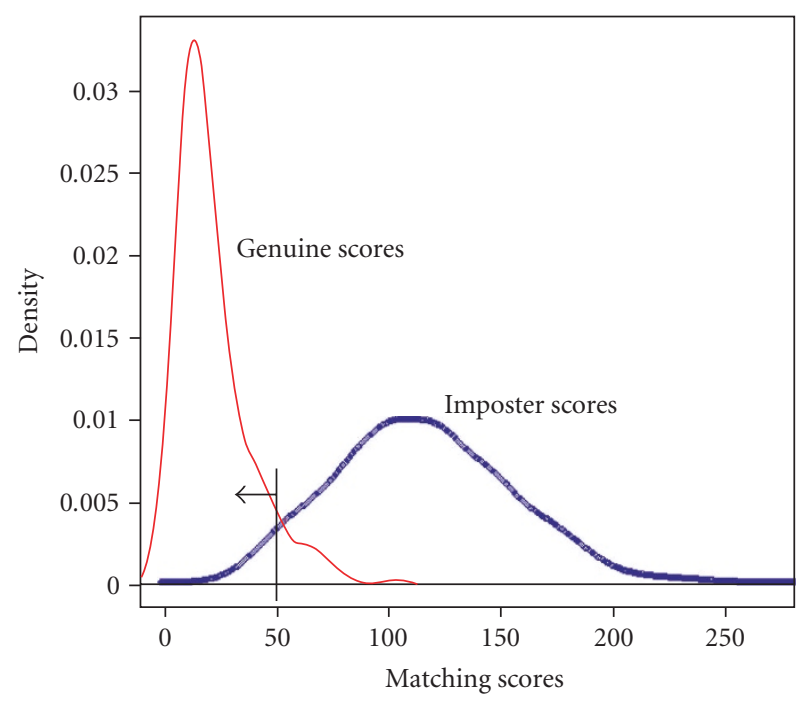

FIgURE 1: Score density plot of biometric device.

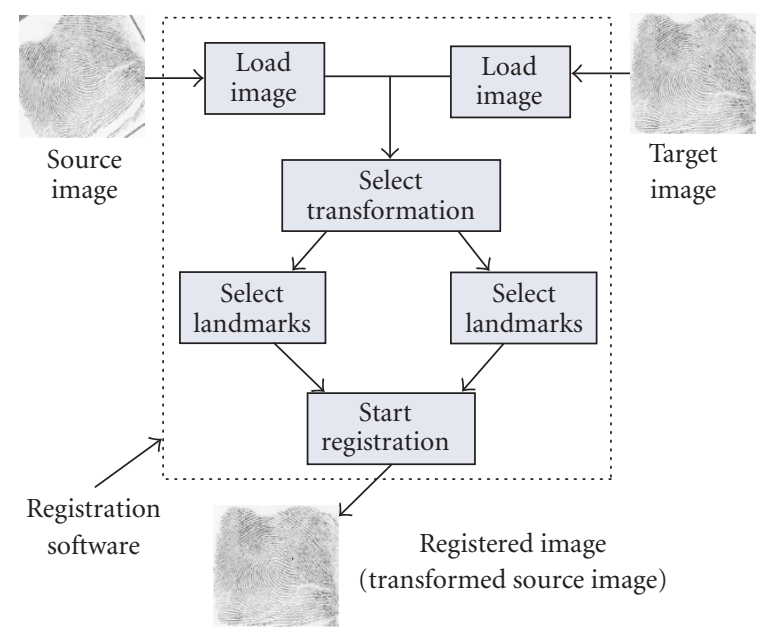

FIgURE 2: The image registration procedure.

position, in order to help a forensic expert in comparing the images and verifying the match.

In order to estimate the reliability of the image registration software module we must take into account its projected operational use and define metrics that evaluate its success during empirical evaluation. The implementation of the image registration algorithm we study is based on the work of Thévenaz et al. We considered their paper [4] as being the informal requirements specification document of the image registration software. We find it rather typical for many image processing systems to be developed without a software requirements specification document. In many cases, even software design documentation is missing or is present in a rudimentary form, far from following the standards common in the software engineering community. This limits the straightforward application of software verification and validation $(\mathrm{V} \& \mathrm{~V})$ standards. The V\&V approach we adopted for this study consists of three steps:

(a) verification of the source code with respect to the requirements by performing code inspections;

(b) validation of the utilized optimization algorithms;

(c) automated reliability testing and failure analysis.

One of the reasons for adopting this approach was to familiarize ourselves with the code and the algorithms, looking for possible implementation errors first. This familiarity, in turn, has been very useful in the process of identifying test cases of particular interest, that is, those that stress the performance of the program and where the algorithm might fail. This approach allowed us to reduce the size of the testing input space and automate the test procedure to achieve greater coverage.

We presented the initial results of our research in [5]. This is an extended version of our earlier work. We expanded the scope of the study by introducing new success metrics used for performance analysis. We also enhanced the failure analysis methodology which is now applicable to a wide range of image processing systems.

The rest of the paper is organized as follows. In Section 2 we define the intended use and calculate the operational profile of the image registration software module. Sections 3 and 4 provide details of the validation methodology, consisting of code inspections and analytical algorithm validation, respectively. Section 5 presents test automation and the reliability estimates we were able to obtain. In Section 6 we describe failure analysis, identifying why the image processing system fails according to the defined metrics. Section 7 concludes the paper.

\section{FINGERPRINT IMAGE REGISTRATION PROCESS}

Figure 2 describes the main steps in the registration process. A forensic expert opens source and target images. He/she selects the type of transformation to be used in the registration process. The available options are scaled rotation or affine transformation. Depending on the transformation chosen, the software asks the user to select two or three landmarks. Landmarks are recognizable physical features in the image, and the user selects them by mouse clicks. In fingerprint images, typical features which can be selected as landmarks are ends of ridges, ridge bifurcations, swirls, or some other characteristic distinguishable points in the image. The same physical features need to be marked in both the source and the target images. User selections are marked on the screen by corresponding cross hairs at manually selected image locations. The cross hairs are color-coded, that is, cross hairs corresponding to the same landmark have the same color in both the source and the target images. Once the selection of landmarks is completed, the user initiates image registration process, which generates a registered image.

Under the assumption that the source and the target images represent the same fingerprint, registration is successful if the landmarks in the registered image are "sufficiently" close to the landmarks in the target image. Successful registration enables a positive match to be established by the expert. However, we had to refine this subjective success metric. 
In consultation with forensic fingerprint experts, we interpreted the success requirement into the following statement: "the distance between the landmarks in the two images (registered and target) must be smaller than the average distance between two ridges in the fingerprint image." So, in order to have the "correct" outcome, the program does not need to produce a "perfect" alignment, but one within a reasonable distance that will not affect the outcome of expert's comparison of the two fingerprint images. Consequently we use the average distance between the landmarks in the registered and target images as a measure of success. This measure has to satisfy some specific threshold which is related to the type of images being processed. In case of fingerprints, for example, we identified this threshold to be the typical distance between the ridges in the image.

Manual selection of landmarks usually introduces human error in the registration process. It is likely that selected landmarks will differ by a few pixels. We expected that this would influence the success rate and reliability of the registration. Based on the expert's opinion, we assumed the following operational profile for user accuracy: positioning within one pixel $-20 \%$ of the time, within 2 pixels $-70 \%$ of the time, within 4 pixels- $10 \%$ of the time.

Another aspect that could influence the success of the registration process is the quality of the image being considered. The degree of self-similarity among the fingerprint images is very high. Therefore, blurred images might cause the alignment optimization algorithm to end up stuck in some local optimum. The probability of this type of failure should decrease in sharp images that clearly depict details.

\section{VERIFICATION BY SOURCE CODE INSPECTION}

The registration process takes two images as inputs, the source and the target, and performs a series of geometric transformations minimizing the pixel differences between them. The goal is to align the source image with the target image. Marquardt-Levenberg (ML) is a well-known general purpose optimization algorithm $[6,7]$. This algorithm is also known to require a significant number of computations and cause long execution times. The developers of the software module under review decided to decrease computation time by adopting a modified Marquardt-Levenberg ( $\left.\mathrm{ML}^{*}\right)$ algorithm, proposed by Thévenaz et al. in [4] for the specific purpose of image registration. As our team was charged with software verification and validation, a point of concern became the numerical optimizations of the ML* algorithm. Therefore, we paid special attention to algorithm validation in the context of the specific usage domain (fingerprint images). Our validation effort consisted of two sets of activities: code inspection $[8,9]$ and algorithm validation. Code inspections are described in this section, algorithm validation in the next section.

\subsection{Specification and implementation cross-validation}

Algorithm 1 provides a brief description of the image registration procedure in the form of a pseudo-code based on the optimized $\mathrm{ML}^{*}$ algorithm [4]. All the equations and symbols used in Algorithm 1 correspond to those in [4]. We conducted very detailed code inspections and compared the code with the specification. One of the reasons for this activity was the need for the members of the validation team to learn and understand the deployed algorithms as well as their implementation. While the cost of detailed inspections is high, we believe it was justified for our project. The consequences of incorrect fingerprint matching in the forensic and security applications are substantial and the prospect of litigation is real. Consequently, eliminating the failed outcomes of the registration algorithm is an imperative.

\subsection{Summary}

Based on the investigation of the specification, literature, and code inspection, we concluded that the image registration module is designed consistently with respect to the claimed references. The transformations it offers are linear and they preserve the essential image features for accurate comparison. We realized that the software package provides implementation of the standard ML algorithm as well as the optimized $\mathrm{ML}^{*}$ algorithm. The $\mathrm{ML}^{*}$ implementation conformed to the algorithm described in [4]. The construction of the Bspline model as well as the pyramidal approach have a sophisticated theoretical basis presented in [10-13]. Through code inspections we did not find any faults in the implementation. While the absence of software faults may surprise some readers, one needs to have in mind that our team served as an independent verification agent. Our activities were intended to go beyond the verification and validation activities performed earlier by the software development organization.

\section{ALGORITHM VALIDATION}

The optimization process is critical for successful image registration. A well-established optimization algorithm and a computationally more efficient modification of the algorithm are both included in the analyzed program. In the core of the registration process is the Marquardt-Levenberg (ML) optimization algorithm. While code analysis activities established correct implementation, in this activity we looked into how ML algorithm was applied, that is, what are the consequences of using this particular optimization algorithm for fingerprint image registration. Another part of this effort was intended to validate that $\mathrm{ML}^{*}$ algorithm, while improving computational efficiency, does not compromise optimization accuracy.

\section{1. $M L$ algorithm validation}

Marquardt-Levenberg (ML) method is frequently used for optimization in nonlinear models (e.g., neural networks, machine learning, machine vision) and has become a virtual standard. To support this claim we note the fact given in [14], stating that Marquardt's original paper [15] is the third most frequently cited paper in all the mathematical sciences.

$\mathrm{ML}$ is a combination of the gradient descent and the Newton optimization method. It is based on fundamental 


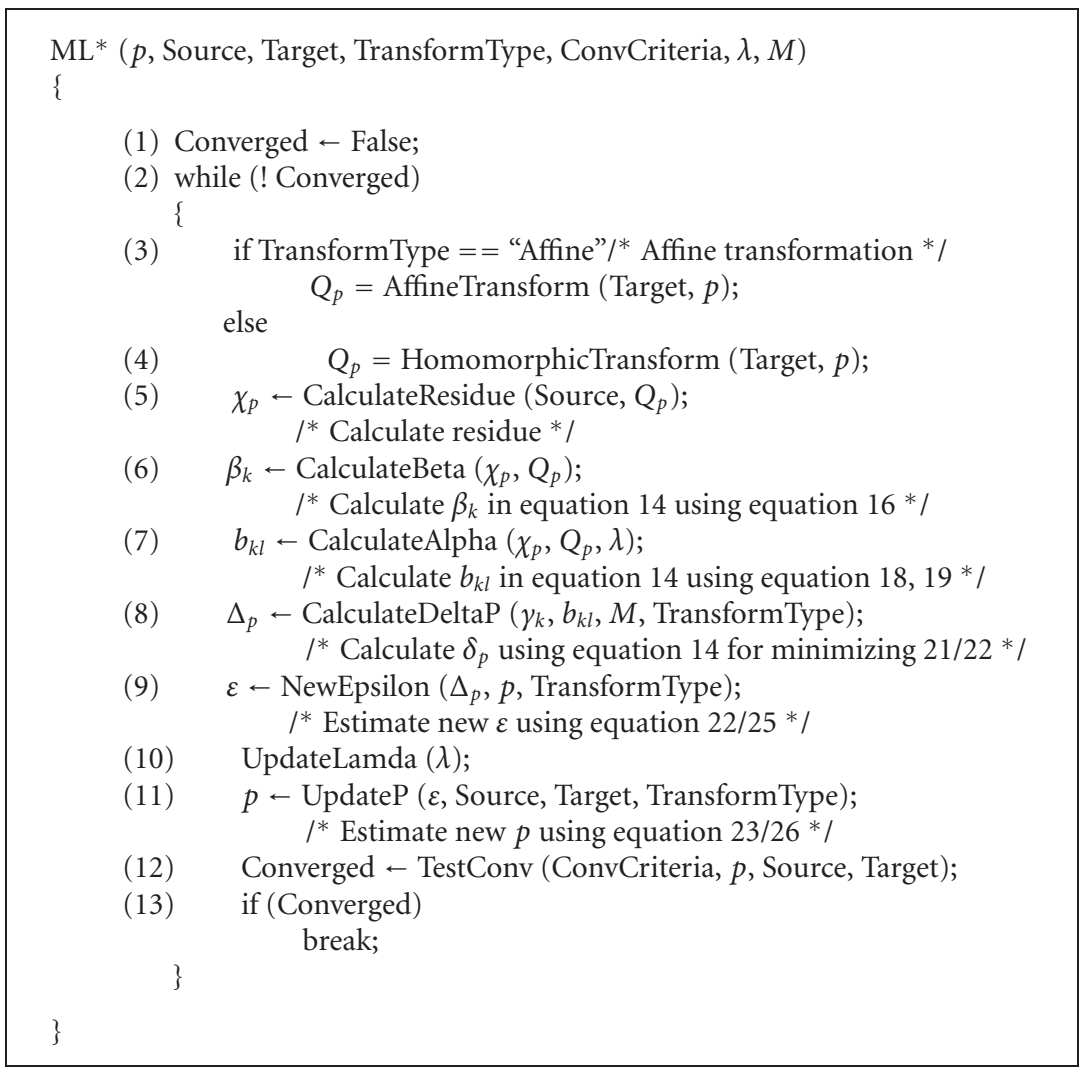

Algorithm 1: Pseudo-code for the ML* algorithm. The equations and symbols correspond to those in [4].

observation that when we are far from the solution the parabolic assumption is wrong so it is better to step along the steepest decent. When we are close to the solution the Newton's step is better.

It is important to understand that this is a heuristic numerical method and that it is not optimal for any welldefined criterion of speed or final error [7]. It represents a well thought out optimization procedure and it works very well in practice. In some special cases [16], the rate of convergence is proved to be quadratic. ML significantly outperforms other nonlinear optimization methods, like gradient descent and conjugate gradient methods, for medium sized problems.

Also it is important to notice that ML does not necessarily find the global optimum. It can become stuck in a local optimum and it may have no ability to escape from it. If we are interested in finding the global optimum, the starting point of the algorithm should be made as close to the optimal point as possible, otherwise it might diverge to some other local optima. The readers should note here the importance that precise placement of landmarks in the initial step of the fingerprint image registration process will have in the results of our analysis.

The only drawback of the ML method is that it requires a matrix inversion step as part of the update, which takes $O\left(n^{3}\right)$ time, where $n$ is the size of the matrix. For medium sized problems this method will be faster than gradient descent plus momentum. However, for large problems, the cost of matrix inversion performed in an inner loop of the algorithm eliminates the quick convergence rates gained by the clever algorithm design.

The authors of $[4,17]$ proposed a modification of the ML algorithm for image registration applications called $\mathrm{ML}^{*}$. They used domain specific knowledge and the structure of the developed nonlinear model to reduce the number of calculations required for single iteration of the algorithm.

The error measure of the source image with respect to the target (or reference) image is defined to be the square of the sum of the pixel intensity differences between the two images:

$$
\begin{gathered}
\mathcal{E}^{2}=\iint_{\{\mathbf{x}\} \subset R^{q}}\left(Q_{\mathbf{p}}\left\{f_{T}(\mathbf{x})\right\}-f_{R}(\mathbf{x})\right)^{2} d \mathbf{x}, \\
\varepsilon^{2}=\left\|Q_{\mathbf{p}}\left\{f_{T}(\mathbf{x})\right\}-f_{R}(\mathbf{x})\right\|^{2},
\end{gathered}
$$

where $f_{R}(\mathbf{x})$ represents the intensity of the pixel at location $\mathbf{x}$ of the reference image, and $Q_{\mathbf{p}}\left\{f_{T}(\mathbf{x})\right\}$ represents the intensity of a pixel which is at the same location after the transformation $Q$ with parameter $\mathbf{p}$.

Although in [4] the authors talk about affine and homomorphic transformations, the actual implementation under analysis [17] contains only the affine case (with two additional subcases: translation and scaled rotation) and bilinear transformation.

Based on our literature review of the ML method, we concluded that the use of the ML method to obtain the optimal parameter $\mathbf{p}$ minimizing (1) is well justified. We would 
like to mention at least the following two relevant points. Fingerprint images are usually $256 \times 256$ pixels large so we should not expect algorithm slowdown due to matrix inversion. Further, precise landmarks can make initial conditions of the optimization problem close to the optimal solution, thus avoiding the local optima problem or the divergence of the method.

For the bilinear case, the code uses the standard ML algorithm for optimization, consequently all that was said about the algorithm (its advantages and disadvantages) holds also for this case. The authors of [4] proposed for the affine cases a modification of the algorithm in order to minimize the number of needed computations. In the next section we will look more closely into this modification.

\subsection{Modified Marquardt-Levenberg algorithm}

In the affine case we have the following two operators: translation operator $T_{\mathbf{b}}$ and an affine operator $A_{\mathbf{A}}$ defined as follows:

$$
T_{\mathbf{b}}(f(\mathbf{x}))=f(\mathbf{x}+\mathbf{b}), \quad A_{\mathbf{A}}(f(\mathbf{x}))=f(\mathbf{A x}) .
$$

So, the combined transformation is

$$
Q_{\mathbf{A}, \mathbf{b}}(f(\mathbf{x}))=f(\mathbf{A} \mathbf{x}+\mathbf{b}) .
$$

In order to minimize (1) the transformation $Q$ is first applied to the source image which is then compared with the reference image. The authors of [4] note that optimizing (1) in the given form requires recalculation of the vector $\left[\beta_{k}\right]$ and the matrix $\left[b_{k l}\right]([7$, equations (16) and (18)] ) because they depend on the transformation parameter $\mathbf{p}=(\mathbf{A}, \mathbf{b})^{\mathrm{T}}$, which changes from iteration to iteration.

Based on the symmetry of the particular transformation of interest (it is equivalent to transform the source image and compare it with the reference image, or to apply inverse transformation to the reference image and then perform the comparison) we can rewrite (1) for the incremental update $\Delta \mathbf{p}=(\Delta \mathbf{A}, \Delta \mathbf{b})^{\mathrm{T}}$ into the following equivalent forms:

$$
\begin{gathered}
\Delta \varepsilon^{2}=\left\|Q_{\mathbf{p}+\Delta \mathbf{p}}\left(f_{T}(\mathbf{x})\right)-f_{R}(\mathbf{x})\right\|^{2}, \\
\Delta \varepsilon^{2}=\left\|Q_{\Delta \mathbf{p}}\left(f_{T}(\mathbf{x})\right)-Q_{-\mathbf{p}}\left(f_{R}(\mathbf{x})\right)\right\|^{2} .
\end{gathered}
$$

In the affine case, minimizing equation (5) with respect to $\Delta \mathbf{p}$ is equivalent to setting $\Delta \mathbf{p}=0$ in (4) and then minimizing the equation with respect to $\mathbf{p}$, which corresponds to the standard ML. However, minimizing equation (5) is more beneficial because the curvature matrix $\left[b_{k l}\right]$ in this case does not depend on the previous value $\mathbf{p}$ and needs to be calculated only once at the parameter value $\Delta \mathbf{p}=0$. The same is true for the partial derivatives $\partial f_{T} / \partial \Delta \mathbf{p}_{\Delta \mathbf{p}=0}$.

We concluded that the proposed modification is mathematically sound and appropriate for the affine case due to the symmetry of applied transformations. Although most probably the paths in the calculation of both methods will be different, we concluded that both algorithms lead to the same optimum, especially in cases where the initial conditions are close to this optimum point. Due to fast convergence of the method we concluded that a significant disparity in the number of iterations between the two algorithms is not expected.

The heuristic and numerical nature of ML and $\mathrm{ML}^{*}$ methods implies that making stronger analytical claims regarding the similarity of their results is not possible. Empirical testing was performed to corroborate the outcome of the analysis.

\subsection{Summary}

$\mathrm{ML}$ is the most frequently used numerical algorithm for nonlinear optimization. It has superlinear rate of convergence observed in practice especially when the first estimate is close to the optimal point. Because of the matrix inversion step, which is required, it makes most sense to apply it to small or medium sized problems. Otherwise, the time required within each iteration grows significantly. By using pyramidal approach the authors address both issues - reducing the problem size in the beginning so that we will get closer to the optimum faster, as well as possibly avoiding local optima, and also harvesting the speed of the method when we are close to the optimal point but the problem size is increased. The proposed $\mathrm{ML}^{*}$ modification for the affine case is mathematically sound and reduces the number of calculations needed. It should give the same results as the original ML algorithm in the fingerprint image registration application, with improved computational efficiency.

However, an important conclusion of this study is that we do not recommend the use of bilinear transformation for identification purposes because of the possible image distortion.

\section{AUTOMATION OF TESTING}

Subsequent to the algorithm analysis and code inspection, we conducted functional performance tests of the image registration system. We developed a methodology to automate the testing as well as tools for test instrumentation and result checking. This section describes the details of the testing process. The code used for testing was TurboReg_.java [17] made available to us by the authors of [4]. This code is used in the fingerprint registration software under review.

We set the following goals for the testing procedure.

(i) Study the accuracy of registration and various transformations when different noise levels are present in images. We call this a "normal case" test. We want to evaluate the impact of image quality on the registration results.

(ii) Study the accuracy of registration when the above conditions apply and the user errs in landmark selection. We call this a "variant case" test. We want to evaluate the impact of user errors on the registration results.

\subsection{Test methodology}

The test methodology we used is presented in Figure 3. First, we select a source image that is to be registered. Then, this image is transformed (scaling, rotation, affine). The 


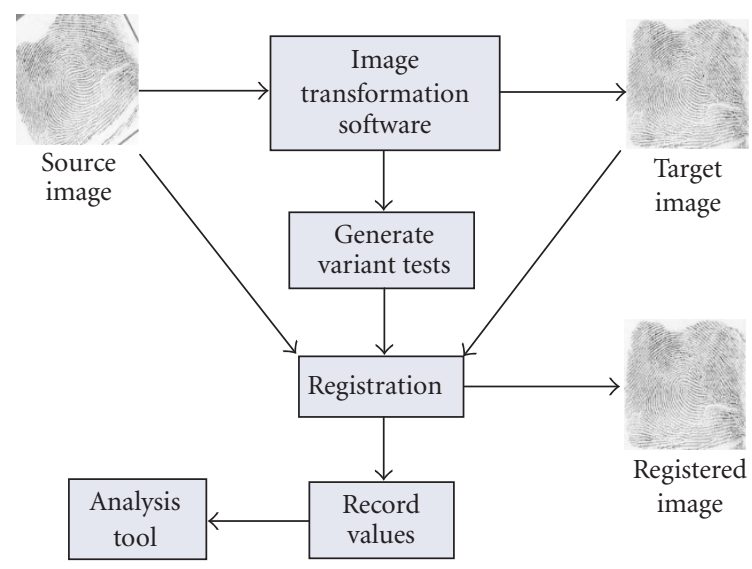

FIgURE 3: Testing methodology.

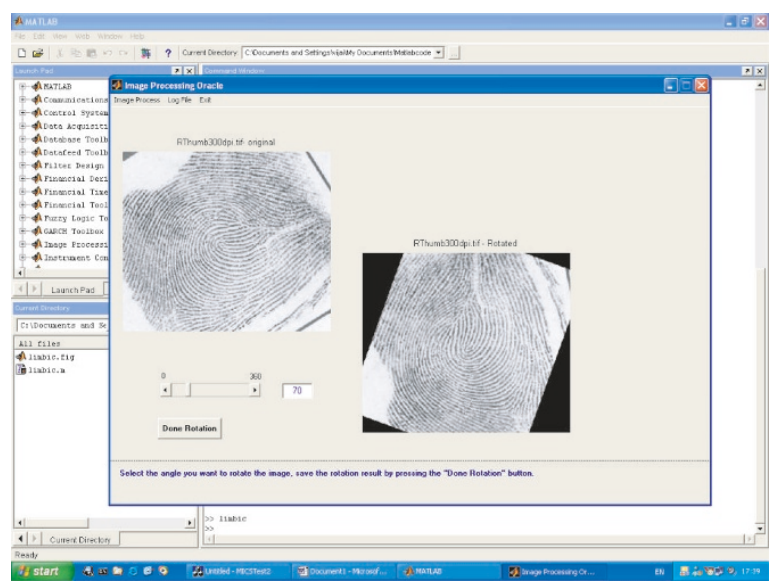

FIgURE 4: Image transformation software developed in MATLAB applies various kinds of transformations and noise on the source image to generate artificial target images.

transformed image will subsequently be used as the target image in the registration process. Figure 4 presents the interface of the image transformation tool, which we developed for the automated generation of tests.

Next, the registration process is performed with the source image and the generated target image. Registration process is monitored and its results are recorded using the automated testing software (ATS), (see Figure 5), another tool we developed during the course of this project. Among other functions, this tool assists testers in generating the parameters for the "variant" test cases. As a reminder, the "variant tests" are those where the user errs by introducing imprecise landmark in the fingerprint images before submitting them to image registration software.

As a final step we perform data analysis to investigate the results of fingerprint image registration program, that is, the difference between the registered and the target images (see Figure 2).

A test on a pair of images (source and target) automatically invokes one "normal test" and four "variant tests." In

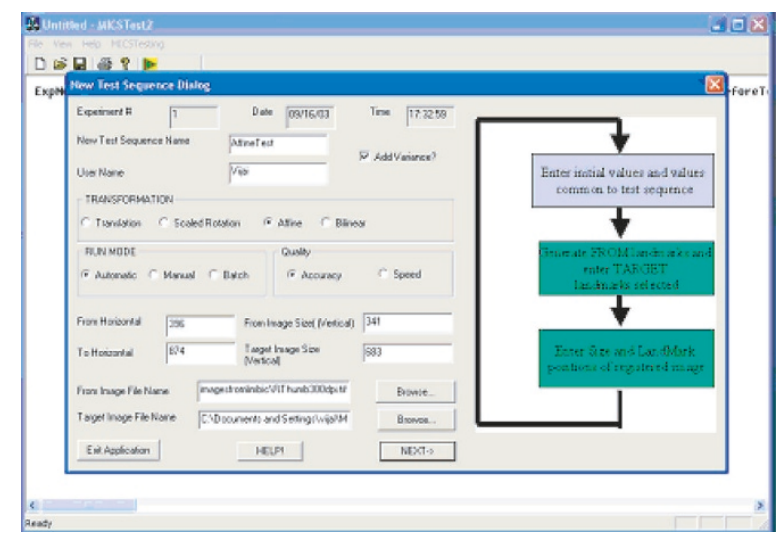

FIGURE 5: Automated testing software.

all tests, we conducted registration following the process described by the software vendor. First a "normal test" which assumes the perfect placement of image landmarks is performed. Then, automated testing software (ATS) modifies the source image landmarks by a small distance; 1,2 , or 4 pixels, in one of the 8 directions (N, NE, E, SE, S, SW, W, $\mathrm{NW}$ ). The simulation of user errors (user-fault-injection) allows us to study how the software responds to inaccurate initial conditions, that is, the imperfect placement of landmarks. For each normal case (source-target image pair) this process is repeated 4 times, with four random user-faultinjection/registration cycles invoked automatically. The selection of landmarks, in terms of the injected errors, follows the operational profile developed earlier and described in Section 2.

To remind readers, based on the expert's opinion, we assumed the following distribution of user accuracy: positioning within one pixel-20\%, within 2 pixels $-70 \%$, within 4 pixels- $10 \%$ of the placement attempts.

Table 1 presents some of the different transformations, user-fault-injection, and noise techniques we used in our testing effort.

\subsection{Results}

We performed tests with source images of different quality. For each source image, we created multiple different target images, as described above. We learned in testing that image quality by itself did not cause the program to fail. Only the execution of the so-called "variant tests" resulted in a few failures. However, image quality combined with the introduced user error and added noise had an impact on failure rates of "variant tests." Therefore, we present below the results of variant tests in different test configurations.

The measure we use for test outcome success determination is the average distance between the landmarks in the image which is the result of the registration algorithm and the target image. We consider the run of the registration program to be successful if the average distance (average error in the position of the landmarks) is smaller than the typical distance between the ridges in the fingerprint image. In 
TABLE 1: Transformations and noise for generation of MATLAB images.

\begin{tabular}{ccccc}
\hline$\#$ & Transform & Noise & Amount & Done \\
\hline 1 & Scaling (S) & None & 1.2 & $\mathrm{Y}$ \\
2 & Rotation (R) & None & 45 & $\mathrm{Y}$ \\
3 & Affine (A) & None & $6,0.2,0 ; 1,6,0 ; 0,0,1 ;$ & $\mathrm{Y}$ \\
4 & $\mathrm{~S}+\mathrm{R}$ & None & $1.2+45$ & $\mathrm{Y}$ \\
5 & $\mathrm{~S}+\mathrm{A}$ & None & $1.2+6,0.2,0 ; 1,6,0 ; 0,0,1 ;$ & $\mathrm{Y}$ \\
6 & $\mathrm{R}+\mathrm{A}$ & None & $45+6,0.2,0 ; 1,6,0 ; 0,0,1 ;$ & $\mathrm{Y}$ \\
7 & $\mathrm{~S}+\mathrm{R}+\mathrm{A}$ & None & $1.2+45+6,0.2,0 ; 1,6,0 ; 0,0,1 ;$ & $\mathrm{Y}$ \\
8 & $\mathrm{~S}$ & Gaussian & 1.2 & $\mathrm{Y}$ \\
9 & $\mathrm{~A}$ & Gaussian & $6,0.2,0 ; 1,6,0 ; 0,0,1 ;$ & $\mathrm{Y}$ \\
10 & $\mathrm{~S}+\mathrm{R}$ & Gaussian & $1.2+45$ & $\mathrm{Y}$ \\
11 & $\mathrm{R}+\mathrm{A}$ & Gaussian & $45+6,0.2,0 ; 1,6,0 ; 0,0,1 ;$ & $\mathrm{Y}$ \\
12 & $\mathrm{~S}+\mathrm{R}+\mathrm{A}$ & Gaussian & $1.2+45+6,0.2,0 ; 1,6,0 ; 0,0,1 ;$ & $\mathrm{Y}$ \\
13 & $\mathrm{~S}$ & Speckle & 1.2 & $\mathrm{Y}$ \\
14 & $\mathrm{~A}$ & Speckle & $6,0.2,0 ; 1,6,0 ; 0,0,1 ;$ & $\mathrm{Y}$ \\
15 & $\mathrm{~S}+\mathrm{R}$ & Speckle & $1.2+45$ & $\mathrm{Y}$ \\
16 & $\mathrm{R}+\mathrm{A}$ & Speckle & $45+6,0.2,0 ; 1,6,0 ; 0,0,1 ;$ & $\mathrm{Y}$ \\
17 & $\mathrm{~S}+\mathrm{R}+\mathrm{A}$ & Speckle & $1.2+45+6,0.2,0 ; 1,6,0 ; 0,0,1 ;$ & $\mathrm{Y}$ \\
18 & $\mathrm{~S}$ & Salt \& pepper & 1.2 & $\mathrm{Y}$ \\
19 & $\mathrm{R}$ & Salt \& pepper & 45 & $\mathrm{Y}$ \\
20 & $\mathrm{~A}$ & Salt \& pepper & $6,0.2,0 ; 1,6,0 ; 0,0,1 ;$ & $\mathrm{Y}$ \\
21 & $\mathrm{~S}+\mathrm{R}$ & Salt \& pepper & $1.2+45$ & $\mathrm{Y}$ \\
22 & $\mathrm{~S}+\mathrm{A}$ & Salt \& pepper & $1.2+6,0.2,0 ; 1,6,0 ; 0,0,1 ;$ & $\mathrm{Y}$ \\
\hline
\end{tabular}

TABLE 2: Test results for low-quality image and scaling/rotation transformations.

\begin{tabular}{cccc}
\hline $\begin{array}{c}\text { Average } \\
\text { user error }\end{array}$ & $\begin{array}{c}\text { Average } \\
\text { registration error }\end{array}$ & $\begin{array}{c}\text { Standard } \\
\text { deviation }\end{array}$ & $\begin{array}{c}\text { Success } \\
\text { rate }\end{array}$ \\
\hline 1 & 1.40 & 0.81 & $100 \%$ \\
2 & 1.54 & 1.24 & $100 \%$ \\
4 & 2.56 & 1.74 & $100 \%$ \\
\hline
\end{tabular}

order to further study test results, we separated these results depending on

(a) the transformation being applied in order to obtain the target image (translation and scaling, affine);

(b) the magnitude of error introduced by the tool in the variance tests.

The following are the results we obtained through experimentation. For lower quality images, we used the threshold of 10 pixels as the acceptable average error. In other words, if the average distance between the landmarks in the aligned image (the result of image registration) and the corresponding target image is less than 10 pixels, the run of the registration program is successful. The distance of 10 pixels was selected because in the fingerprint images that we used for testing, the closest ridges were never less than 12 pixels apart. Consequently, a fingerprint analysis expert can correctly interpret an error of up to 10 pixels. The rest of this section presents test results.
TABLE 3: Test results for low-quality image and affine transformations.

\begin{tabular}{cccc}
\hline $\begin{array}{c}\text { Average } \\
\text { user error }\end{array}$ & $\begin{array}{c}\text { Average } \\
\text { registration error }\end{array}$ & $\begin{array}{c}\text { Standard } \\
\text { deviation }\end{array}$ & $\begin{array}{c}\text { Success } \\
\text { rate }\end{array}$ \\
\hline 1 & 4.34 & 3.38 & $91.18 \%$ \\
2 & 4.88 & 4.85 & $95.45 \%$ \\
4 & 6.34 & 7.07 & $89.47 \%$ \\
\hline
\end{tabular}

TABLE 4: Test results for high-quality image and affine transformations.

\begin{tabular}{cccc}
\hline $\begin{array}{c}\text { Average } \\
\text { user error }\end{array}$ & $\begin{array}{c}\text { Average } \\
\text { registration error }\end{array}$ & $\begin{array}{c}\text { Standard } \\
\text { deviation }\end{array}$ & $\begin{array}{c}\text { Success } \\
\text { rate }\end{array}$ \\
\hline 1 & 2.90 & 2.34 & $100 \%$ \\
2 & 4.14 & 2.69 & $100 \%$ \\
4 & 3.44 & 2.33 & $91.67 \%$ \\
\hline
\end{tabular}

With no failures observed (Table 2), the experimentally obtained reliability measure for low-quality images and scaling/rotation transformations only is $100 \%$. In this paper, the reliability is defined as the proportion of program executions that result in a successful fingerprint image registration.

Our next set of tests used the images of the same quality as above, but this time we applied affine transformations. The results of these tests are shown in Table 3 . When we apply the operational profile of $(0.2,0.7,0.1)$, which describes the typical distribution of errors in the placement of the landmarks, experimental reliability for this operational mode is estimated to be approximately $94 \%$.

The second set of experiments was performed with images of better quality. Same as in the operational scenarios with lower quality images, we used the threshold of 10 pixels as an indication of registration success. The following is the list of our results.

Similar to the outcome of the experiments with the lowquality image case, image translations and rotations did not cause any failures of the registration program. The reliability in this operational mode was estimated to be $100 \%$.

We also tested high-quality images in combination with affine transformations. The results of these tests are shown in Table 4. By applying the operational profile of $(0.2,0.7$, 0.1 ) as weighting factors in the linear combination of success rates from Table 4, experimental reliability for this operational mode is approximately $99.17 \%$.

\section{FAILURE ANALYSIS}

By using the defined metrics for success, which were validated by the domain experts, the testing process provided evidence supporting our hypotheses about the robust performance of the image registration system reached in the source code validation and algorithm validation analyses. Based on all three steps of our methodology we identified that the success rate of the fingerprint image registration software module depends on the following three parameters. 
(a) User errors introduced in the selection of the landmarks. Small errors make the optimization algorithm's initial state very close to the optimal solution, thus reducing the possibility of getting trapped in a local optimum.

(b) The types of transformations used in the generation of image distortions, which mimic real-world latent fingerprint images. Complex transformations, such as affine, combined with the user errors in marking landmarks caused several system failures. We were able to trace these failures to the issue of self-similarity of the fingerprint images which guided the algorithm to a nonoptimal solution.

(c) The quality of the images, while not the determining factor per se, had an impact on observed failures. Better quality images provide crisper information to the optimization algorithm which, in turn, avoids being trapped in a local optimum.

One suggestion for improvement of the fingerprint registration system is to investigate the application of other optimization algorithms that can avoid local minima entrapment at the expense of being computationally more expensive. These algorithms could improve the reliability results obtained in our experiments.

\section{CONCLUSIONS}

The increased use of biometric systems requires additional research efforts related to their reliability estimation. However, the reliability prediction of biometric systems is not the only open assessment problem, as verification and validation standards for image processing systems are not well defined either. We were asked to validate a module of a commercially available system used in fingerprint analysis, the fingerprint registration software. Due to concerns about proprietary information, this paper does not reveal the product identity. However, we believe that the experiences reported here are sufficiently generic and applicable to verification and validation of similar image registration/processing systems.

Our approach towards validation and reliability estimation consisted of three steps:

(a) validation of the source code with respect to the system requirements specification;

(b) validation of the optimization algorithm, which is in the core of the registration system;

(c) automation of testing.

Source code verification provided evidence that the system has been implemented right with respect to the research paper describing its technical requirements. Further, it provided insights into the actual design of the software implementation. Through algorithm validation we were able to draw conclusions about the expected performance of the system. In principle, this step corresponds to requirements validation step in traditional software engineering literature. Furthermore, the outcomes of the analysis allowed us to specify interesting test cases and operational modes that indicated the limits of robustness of the system under test.
Testing provided further evidence corroborating the conclusions reached in the previous steps.

We consider this study an early attempt to define processes for the verification and validation of biometric technologies. As biometric systems continue to play increasingly important role in user authentication, homeland security, military and forensic applications, similar studies will be needed to further our ability to reason about system and software reliability prior to deployment.

\section{REFERENCES}

[1] A. K. Jain and S. Pankanti, "Automated fingerprint identification and imaging systems," in Advances in Fingerprint Tech$n o \log y$, H. C. Lee and R. E. Gaensslen, Eds., CRC Press, Boca Raton, Fla, USA, 2nd edition, 2001.

[2] A. K. Jain, A. Ross, and S. Prabhakar, "Fingerprint matching using minutiae and texture features," in Proceedings of International Conference on Image Processing (ICIP '01), vol. 3, pp. 282-285, Thessaloniki, Greece, October 2001.

[3] S. Prabhakar, J. Wang, A. K. Jain, S. Pankanti, and R. Bolle, "Minutiae verification and classification for fingerprint matching," in Proceedings of the 15th International Conference on Pattern Recognition (ICPR '00), vol. 1, pp. 25-29, Barcelona, Spain, September 2000.

[4] P. Thévenaz, U. E. Ruttimann, and M. Unser, "A pyramid approach to subpixel registration based on intensity," IEEE Transactions on Image Processing, vol. 7, no. 1, pp. 27-41, 1998.

[5] D. Desovski, V. Gandikota, Y. Liu, Y. Jiang, and B. Cukic, "Validation and reliability estimation of a fingerprint image registration software," in Proceedings of the 15th International Symposium on Software Reliability Engineering (ISSRE '04), pp. 306-313, Bretagne, France, November 2004.

[6] W. H. Press, B. P. Flannery, S. A. Teukolsky, and W. T. Vetterling, Eds., Numerical Recipes in C: The Art of Scientific Computing, Cambridge University Press, Cambridge, UK, 1988-1992.

[7] S. Roweis, "Levenberg-Marquardt Optimization," http://www. cs.toronto.edu/ roweis/notes.html.

[8] J. K. Chaar, M. J. Halliday, I. S. Bhandari, and R. Chillarege, "In-process evaluation for software inspection and test," IEEE Transactions on Software Engineering, vol. 19, no. 11, pp. 10551070, 1993.

[9] M. S. Fisher and B. Cukic, "Automating techniques for inspecting high assurance systems," in Proceedings of the 6th IEEE International Symposium on High Assurance Systems Engineering (HASE '01), pp. 117-126, Boco Raton, Fla, USA, October 2001.

[10] M. Unser, "Splines: a perfect fit for signal and image processing," IEEE Signal Processing Magazine, vol. 16, no. 6, pp. 22-38, 1999.

[11] M. Unser, A. Aldroubi, and M. Eden, "B-spline signal processing. I. Theory," IEEE Transactions on Signal Processing, vol. 41, no. 2, pp. 821-833, 1993.

[12] M. Unser, A. Aldroubi, and M. Eden, "B-spline signal processing. II. Efficiency design and applications," IEEE Transactions on Signal Processing, vol. 41, no. 2, pp. 834-848, 1993.

[13] M. Unser, A. Aldroubi, and M. Eden, "The $L_{2}$-polynomial spline pyramid," IEEE Transactions on Pattern Analysis and Machine Intelligence, vol. 15, no. 4, pp. 364-379, 1993.

[14] comp.ai.neural-nets FAQ, http://www.faqs.org/faqs/ai-faq/ neural-nets/. 
[15] D. W. Marquardt, "An algorithm for least-squares estimation of nonlinear parameters," SIAM Journal on Applied Mathematics, vol. 11, pp. 431-441, 1963.

[16] N. Yamashita and M. Fukushima, "On the rate of convergence of the Levenberg-Marquardt method," Computing, no. [Suppl] 15, pp. 227-238, 2001.

[17] Turbo Registration plug-in, http://bigwww.epfl.ch/thevenaz/ turboreg/.

Dejan Desovski is a Ph.D. candidate in computer science at West Virginia University, USA. He obtained his B.S. degree in computer science from Ss. Cyril and Methodius University in Skopje, Republic of Macedonia, and his M.S. degree from West Virginia University. His research interests include software V\&V, combining formal methods and testing, and software reliability analysis and estimation.

Vijai Gandikota received the B.E. degree in electronics and communications engineering from Andhra University, India, and the M.S. degree in electrical engineering from West Virginia University, and is currently pursuing the M.S. degree in computer science at West Virginia University. $\mathrm{He}$ is presently a software engineer with IBM Inc. His interests are in the areas of software design and development, software $\mathrm{V} \& \mathrm{~V}$, machine learning, and fractals.

Yan Liu received the B.S. degree in computer science from Wuhan University, China, and the M.S. and Ph.D. degrees in computer science from West Virginia University. She is currently a research scientist at Motorola Labs, Motorola Inc. Her research interests are in the areas of software $\mathrm{V} \& \mathrm{~V}$, machine learning, and statistical learning.

Yue Jiang received the B.S. degree in electrical engineering from Changchun Technology University, China, and the M.S. degree in computer science from West Virginia University. She is currently a Ph.D. student in West Virginia University. Her research interests are in the areas of software $\mathrm{V} \& \mathrm{~V}$, machine learning, and bioinformatics.

Bojan Cukic is an Associate Professor in the Lane Department of Computer Science and Electrical Engineering at West Virginia University, where he also serves as a Codirector of the Center for Identification Technology Research. His research interests include software engineering for highassurance systems, fault-tolerant computing, information assurance, and biometrics.
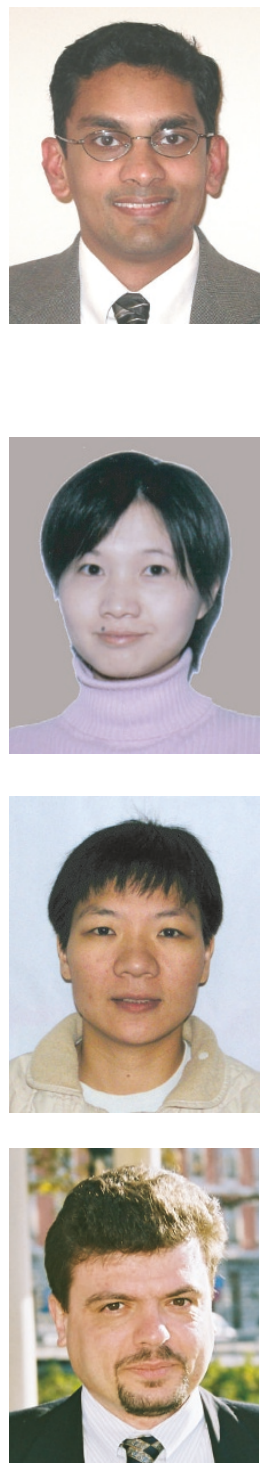

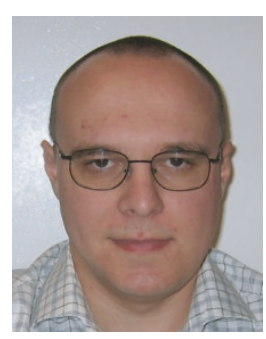

He received a US National Science Foundation Career Award and a Tycho Brahe Award for research excellence from NASA Office of Safety and Mission Assurance. He received his Ph.D. degree in computer science from the University of Houston. 